\title{
CAMBIOS EN LA SINTOMATOLOGÍA MICCIONAL DE LA INCONTINENCIA URINARIA DE ESFUERZO TRAS LA CIRUGÍA CON TÉCNICAS DE CABESTRILLO TRANSVAGINAL.
}

\author{
Benjamín Martínez Córcoles, Antonio S. Salinas Sánchez, Miguel Segura Martín, José Miguel \\ Giménez Bachs, María José Donate Moreno, Héctor Pastor Navarro, Pedro Carrión López \\ María Ángeles Valero Álvarez', Clotilde Fernández Olano' y Julio Antonio Virseda Rodríguez.
}

Servicio de Urología. Complejo Hospitalario Universitario de Albacete. 'Gerencia de Atención Primaria de Albacete. España.

Resumen.- OBJETIVO: Evaluar los cambios en la sintomatología miccional de las pacientes con incontinencia urinaria de esfuerzo (IUE), intervenidas con técnicas de cabestrillo transvaginal, durante un periodo de cinco años en el Servicio de Urología del Complejo Hospitalario Universitario de Albacete (CHUA).

MÉTODO: Entre noviembre de 2001 y diciembre de 2005, se han intervenido en nuestro centro a 126 pacientes (36-78; media 57,09 años) afectas de IUE, mediante técnicas de cabestrillo transvaginal (Técnicas de Sling In Fast, TVT y TOT). Todas las pacientes fueron evaluadas mediante clínica y urodinamia.

RESULTADOS: El índice de masa corporal (IMC) medio fue de 28, $14 \mathrm{Kg} / \mathrm{m} 2$ (DE: 4,66; IC 95\%: 27,3228,96). 92 pacientes (73\%) presentaban entre 2 y 4 embarazos. 99 pacientes $(80,9 \%)$ presentaban entre 2 y 4 partos. Todos los partos fueron vaginales a excepción de 12 casos $(9,5 \%)$ en que se practicó cesárea. Tras la cirugía la frecuencia miccional diurna fue superior a 120 minutos en 112 pacientes $(88,9 \%)$, con respecto a la nocturna 110 pacientes $(87,3 \%)$, se levantaban por la noche a orinar menos de 2 veces. 104 pacientes $(82,5 \%)$ presentaban menos de 2 episodios de escape a día y 105 pacientes (83,3\%) necesitaban como mucho usar una compresa al día durante la última semana tras la cirugía. Disminuyó en número de escapes al día tras la cirugía en 114 pacientes (90,5\%), con una disminución media de 9,65 escapes (IC: 95\%; $8,56-10,79)(p<0,0001) .94$ pacientes $(76,4 \%)$ estaban totalmente secas.

CONCLUSIONES: El desarrollo de nuevas técnicas quirúrgicas para el tratamiento de la IUE, ha favorecido los resultados y ha disminuido el número de complicaciones, manifestándose en la evolución favorable de los cambios miccionales tras la cirugía.

Palabras clave: Incontinencia urinaria de esfuerzo. Síntomas miccionales. Cirugía.

Summary.- OBJECTIVES: To evaluate the changes in voiding symptoms in female patients with stress urinary incontinence (SUI) undergoing transvaginal sling techniques over a five-year period in the Department of Urology at the University Hospital of Albacete.

METHODS: Between November 2001 and December 2005126 patients with SUI (mean age 57.09 years; 36-78) underwent transvaginal sling techniques (Sling in 
Fast, TVT, TOT). All patients were evaluated clinically and urodynamically.

RESULTS: Average body mass index (BMI) was 28.14 $\mathrm{kg} / \mathrm{m} 2$ (SD 4.66; 95\% Cl: 27.32-28.96). 92 patients (73\%) presented between 2-4 previous pregnancies. 99 patients (80.9\%) have had birth labour between 2 and 4 times. All of them were vaginal birth labours except 12 cases (9.5\%) in which caesarean section had been performed. Daytime voiding frequency after surgery was over 120 minutes in 112 patients (88.9\%). Night-time voiding frequency was equal or less than twice in 110 patients (87.3\%). 104 patients (82.5\%) presented at least two leaking episodes per day, and 105 patients $(83.3 \%$ ) needed to wear one pad per day or less during the last week before follow-up visit. The number of urinary leak episodes per day diminished in 114 patients $(90.5 \%$ ) with a mean decrease of 9.65 episodes ( $95 \% \mathrm{Cl}: 8.56-10.79$ ) (p<0 0.0001$)$. Ninety-four patients $(76.4 \%$ ) were completely dry .

CONCLUSIONS: Development of new surgical techniques for the treatment of SUI have improved results and diminished the number of complications, an expression of which is the favourable evolution of voiding changes after surgery.

Keywords: Stress urinary incontinence. Voiding symptoms. Surgery.

\section{INTRODUCCIÓN}

En 1979, Bates y cols. definieron la Incontinencia Urinaria (IU) como la pérdida involuntaria de orina a través de la uretra, que se puede demostrar de forma objetiva y que ocasiona un problema social o de higiene (1). En 2002, la International Continence Society (ISC) actualizó la normalización terminológica y las definiciones de la función del tracto urinario inferior (TUI), para poder acordar y utilizar después términos y definiciones idénticos y uniformes de los estados asociados con una disfunción del tracto urinario inferior (DTUI), como la IU (2), definiendo la incontinencia urinaria de esfuerzo (IUE) como la "pérdida involuntaria de orina que tiene lugar cuando, en ausencia de contracción del detrusor, la presión intravesical supera la presión uretral máxima".

La IU, puede ser el resultado de una disfunción vesical o esfinteriana aunque existen algunos factores, que por diversos mecanismos, predisponen a la aparición de la IU y, en algunos casos, a agravarla. Entre ellos destacan: la diabetes, la obesidad, la depresión, la caquexia, la menopausia y el envejecimiento.
La IU es un problema que afecta aproximadamente al $6 \%$ de la población estadounidense y aunque se estima una prevalencia en los adultos del 15 al 30\%, se presenta en todas las edades, detectándose un incremento progresivo según avanza la edad. Así mismo supone un gran coste, estimado en 10 billones de dólares al año (3).

En España está reconocido como un problema de salud que genera un gran gasto económico a los sistemas sanitarios públicos. En el Área 6 de Madrid, en el año 2000, supuso el $8,8 \%$ del gasto total de farmacia (4).

La IUE es un síntoma que refiere la paciente, un signo objetivable con el incremento de presión intraabdominal y una condición urodinámicamente demostrable que señala una alteración de la fase de llenado vesical $(1,2,5)$.

El gold estándar del tratamiento de la IUE es el tratamiento quirúrgico que está indicado en pacientes con importante pérdida de orina, para las que su incontinencia supone un problema social e higiénico. La mayoría de intervenciones ideadas para tratar la IUE tienen como finalidad elevar la unión uretrovesical restableciendo así su relación anatómica con el pubis de manera que la uretra posterior quede en situación suprapúbica para permitir que los incrementos de presión abdominal se transmitan a la uretra (6). Tras las iniciales técnicas de cirugía vía abdominal, fueron apareciendo otras técnicas vía vaginales, hasta las más utilizadas actualmente consistentes en la colocación vía vaginal de cabestrillos: cabestrillo suburetral con fascia de los rectos. (MC-GUIRE (7)), tensión-free Vaginal Tape (TVT (8)), cabestrillo transobturatriz (TOT $(9,10))$, regulador mecánico externo (REMEEX (11)) ó técnicas de anclaje óseo (IN FAST $(12,13)$ ). Actualmente están en desarrollo técnicas que utilizan células madre (14).

Aunque el patrón de referencia en cuanto a los resultados de estas técnicas ha sido la técnica de Marshall-Marchetti-Krantz, los datos publicados hasta el momento con técnicas de cabestrillo transvaginal sugieren un importante avance en cuanto a la mejoría de la IUE. Presentamos los resultados obtenidos en nuestro Servicio en cuanto a los cambios en la sintomatología miccional en pacientes con IUE intervenidas con este tipo de técnicas.

\section{MATERIAL Y MÉTODO}

Se trata de un estudio cuasi-experimental de un solo grupo. Se analizan los resultados obtenidos en sujetos en lista de espera para ser intervenidas por IUE 
y posteriormente, seis meses después de la intervención. Las pacientes fueron diagnosticadas de IUE e intervenidas quirúrgicamente en el Servicio de Urología del Complejo Hospitalario Universitario de Albacete. El estudio se inició en noviembre de 2001 y finalizó en diciembre de 2005 y mediante muestreo consecutivo se seleccionaron un total de 126 pacientes.

Se analizó la sintomatología miccional en relación a:

- Tiempo de evolución de la incontinencia (en meses).

- Tratamientos médicos de la IU.

- Tipos de tratamientos médicos de la IU.

- Maniobras de Kegel.

- Antecedentes de ITU.

- Frecuencia miccional diurna (en minutos).

- Frecuencia miccional nocturna (número de veces).

- Número de episodios de escapes al día en la última semana.

- Número de compresas que usa al día.

- Presencia de cistocele.

- Grado de cistocele: I-II-III. Grado I: Existe mínima hipermovilidad de la base de la vejiga. Grado II: La base de la vejiga protuye en el introito sin exteriorizarse. Grado III: La vejiga protuye fuera de la vagina con el esfuerzo.

- Rectocele.

- Urgencia miccional.

- Incontinencia de urgencia.

- Informe urodinámico: urodinámica normal, incontinencia pura, incontinencia mixta.

Se recogieron datos relacionados con la intervención quirúrgica, incluyendo el preoperatorio y la propia intervención:

- Riesgo quirúrgico /Clasificación de la Sociedad Americana de Anestesiología: desde el nivel I -riesgo mínimo- a nivel $\mathrm{V}$-pacientes moribundos-).

- Pruebas de función renal: urea y creatinina.

- Tipo de cirugía practicada: Sling In Fast, TVT, TOT.

- Cirugía asociada: colporrafia anterior (Kelly), colporrafia posterior, histerectomía.

- Existencia de complicaciones peroperatorias.

- Existencia de complicaciones postoperatorias inmediatas.

- Días de sonda.

Además se analizaron: edad, índice de masa corporal, antecedentes de cirugía abdominal, presencia de enfermedades crónicas, consumo de medicación crónica referido por los pacientes y antecedentes obstétricos: número de partos por vía vaginal o cesárea, número de embarazos, número de abortos, antecedente de histerectomía. (vía vaginal, abdominal o radical), tratamientos con estrógenos.
La valoración inicial se realizó después de sentar la indicación quirúrgica a la paciente estudiada en la consulta de Urología de nuestro Hospital. La segunda valoración (postest) se realizó seis meses después de la intervención previa citación, analizándose:

- Episodios de ITU.

- Frecuencia miccional diurna (en minutos).

- Frecuencia miccional nocturna (numero de veces).

- Episodios de escape al día en la última semana.

- Número de compresas que usa al día.

- Cistocele.

- Grado de cistocele.

- Rectocele.

- Complicaciones tardías.

Se realizó un análisis descriptivo de cada una de las variables del estudio además de un análisis bivariante mediante pruebas de independencia para comparar proporciones observadas en grupos independientes (chi-cuadrado), y cuando no se cumplieron las condiciones de aplicación se empleó la prueba exacta de Fisher. En la comparación de proporciones observadas en los mismos sujetos, mediante medidas repetidas, se empleó la prueba de simetría de McNemar y en el caso de una variable binaria y otra con más de dos categorías ordenadas, se aplicó la prueba de tendencia lineal de MantelHaenscel.

Para estudiar la relación entre una variable binaria y una cuantitativa, se utilizó la prueba " $\mathrm{t}$ " de Student-Fisher y se utilizaron pruebas no paramétricas (U de Mann-Whitney en grupos independientes y $\mathrm{T}$ de Wilcoxon en medidas repetidas) cuando la distribución de alguna de las variables no cumplía las condiciones de aplicación. En la comparación de medias en más de dos grupos independientes se utili-

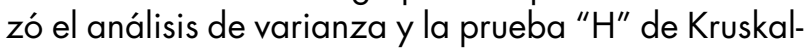
Wallis.

\section{RESULTADOS}

La edad media de las 126 mujeres de la muestra fue de 57,09 años con una desviación típica (DE) de 9,57 y un intervalo de confianza al $95 \%$ (IC 95\%) comprendido entre 55,40 y 58,78 . La edad mínima fue de 36 años y la máxima de 76 años. El grupo de edad más frecuente correspondió a las pacientes comprendidas entre los 56 y 60 años $(20,6 \%$. El 77,6\% de las mujeres de la muestra presentaron edades comprendidas entre los 46 y los 65 años. En la Figura 1 se muestra la proporción de cada grupo de edad por intervalos. 




FIGURA 1.Distribución de las pacientes por grupo de edad.

Manifestaron padecer alguna enfermedad de carácter crónico y no relacionada con su patología urológica 88 pacientes $(69,8 \%$ ). El 42,1\% de las mujeres presentaron solo una enfermedad 153 casos), mientras que únicamente 2 de ellas $(1,6 \%)$ refirieron 4 enfermedades crónicas.

Las pacientes con alguna enfermedad crónica presentaron una edad media superior a las que no se declararon enfermas: 58,47 frente a 53,89 años $(p=0,013)$. En la Tabla I se describen los problemas de salud excluyendo los problemas urológicos, declarados por las pacientes o comprobadas en sus historias clínicas siguiendo la clasificación CIPSAP-2 Definida.

Las enfermedades del aparato circulatorio fueron las más frecuentes $(47,72 \%)$, seguidas de las endocrinas, nutricionales o metabólicas $(43,18 \%)$.
La hipertensión arterial, complicada o no, fue la enfermedad más frecuente declarada por las pacientes $(39,77 \%)$, seguida de la artrosis $(27 \%)$ y diabetes $(25 \%)$.

Estaban tomando algún tipo de medicación no relacionado con tratamientos médicos de su incontinencia urinaria 85 mujeres $(67,5 \%)$. El 40,5\% de las mujeres utilizaban un solo fármaco $151 \mathrm{mu}$ jeres), mientras $8(9,5 \%)$ necesitaban 3 ó 4 medicamentos. A mayor número de enfermedades crónicas presentes, el número de fármacos utilizados fue mayor $(p<0,0001)$. Los fármacos más frecuentemente utilizados fueron los hipotensores $(41,18 \%)$ seguido de los antiinflamatorios no esteroideos (AINEs) $(27,06 \%)$. En la Tabla II se describe la distribución de frecuencias de los fármacos utilizados por las pacientes del estudio.

El índice de masa corporal (IMC) medio fue de 28, $14 \mathrm{Kg} / \mathrm{m} 2$ (DE: 4,66; IC 95\%: 27,32-28,96), con un rango entre 20,03 y $42,87 \mathrm{Kg} / \mathrm{m} 2$. Un $42,9 \%$ de las mujeres (54 casos) presentaron sobrepeso (IMC entre 25 y $30 \mathrm{Kg} / \mathrm{m} 2)$ y un $30,2 \%$ (38 casos) presentaron un IMC en rango de obesidad (> $30 \mathrm{Kg} / \mathrm{m} 2$ ). En la Figura 2 se muestra la distribución de las pacientes según su IMC.

La edad media de la menarquia fue de 12,09 años (DE: 1,3; IC 95\%: 11,22-12,96) con rango entre los 10 y 14 años. En el momento del estudio, 96 mujeres $(76,2 \%)$ estaban menopausicas, con una edad media de inicio de ésta a los 46,09 años (DE: 5,34 ; IC 95\%: $42,51 \%-49,68$ ) con rango entre los 37 y 53 años. La edad de menarquia y la de menopausia no se correlacionaron significativamente.

\section{TABLA I. PROBLEMAS DE SALUD DE LOS PACIENTES (CIPSAP-2 DEFINIDA).}

\begin{tabular}{|l|c|}
\hline PROBLEMA DE SALUD & $\mathbf{N}(\%)^{*}$ \\
\hline III. Enfermedades endocrina, nutricionales, metabólicas & $38(43,18)$ \\
\hline V. Enfermedades mentales & $19(21,59)$ \\
\hline VII. Enfermedades del aparato circulatorio & $42(47,72)$ \\
\hline VIII. Enfermedades del aparato respiratorio & $3(3,4)$ \\
\hline IX. Enfermedades del aparato digestivo & $1(1,36)$ \\
\hline XIII. Enfermedades del aparato locomotor y tejido conectivo & $32(36,36)$ \\
\hline
\end{tabular}


TABLA II. TIPOS DE FÁRMACOS UTILIZADOS.

\begin{tabular}{|l|c|}
\hline & $\mathbf{N}(\%)^{*}$ \\
\hline HIPOTENSORES & $35(41,18)$ \\
\hline AINES & $23(27,06)$ \\
\hline ANTIDEPRESIVOS & $19(22,35)$ \\
\hline HIPOLIPEMIANTES & $19(22,35)$ \\
\hline ANTIDIABÉTICOS & $12(14,12)$ \\
\hline VASODILATADORES & $8(9,41)$ \\
\hline BIFOSFONATOS & $8(9,41)$ \\
\hline HORMONAS TIROIDEAS & $8(9,41)$ \\
\hline ANTIASMÁTICOS & $3(3,53)$ \\
\hline
\end{tabular}

Un $73 \%$ de la muestra (92 casos) presentaron entre 2 y 4 embarazos, existiendo únicamente 2 pacientes que no habían estado nunca embarazadas $(1,6 \%)$, mientras que 3 tuvieron hasta 9 embarazos $(2,4 \%)$. Hubo un total de 37 abortos $(29,4 \%)$.

Por lo que respecta al número de partos, un $80,9 \%$ de las mujeres tuvieron entre 2 y 4 partos 199 casos), mientras que dos mujeres fueron nulíparas $(1,6 \%)$ y 2 tuvieron 9 partos $(1,6 \%)$. Todos los partos fueron vaginales a excepción de 12 casos $(9,5 \%)$ en los que hubo que practicar cesárea en una $\circ$ más ocasiones. En la Tabla III se resumen el número de embarazos, partos, cesáreas y abortos que presentaron las pacientes del estudio.

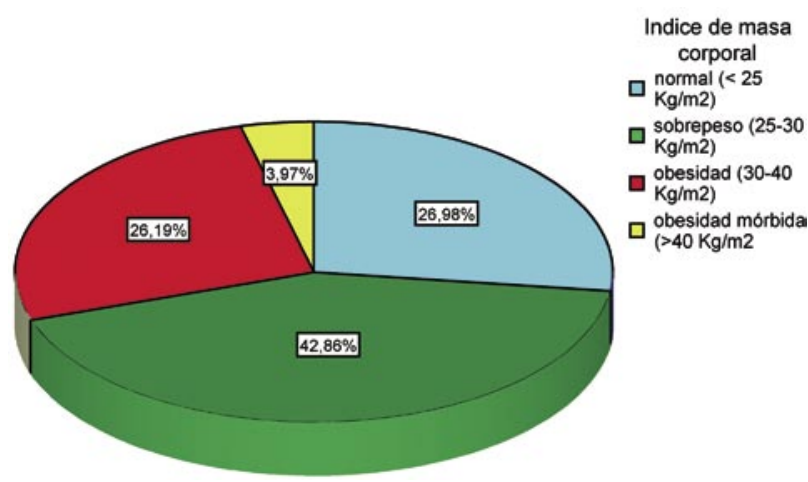

FIGURA 2. Distribución de las pacientes según su IMC.
Tuvieron antecedentes de histerectomía 32 mujeres $(25,4 \%)$, realizándose ésta por vía abdominal en $26(81,25 \%)$ y en el resto de casos por vía vaginal. La edad media a la que se realizó la histerectomía fue 49,71 años (DE: 8,97; IC 95\%: 41,88$53,94)$. No se encontraron diferencias significativas en cuanto a la media de edad de las pacientes que estaban $\circ$ no histerectomizadas. Por otro lado, 13 pacientes $(10,3 \%)$ habían sido sometidas a doble anexectomía, a una edad media de 48,82 años (DE: 8,59; IC 95\%: 43,05-59,59). Finalmente, 7 pacientes $(5,6 \%)$ llevaban tratamiento sustitutivo con estrógenos $(5$ por vía transepidérmica y 2 por vía oral).

Existieron antecedentes de cirugía por incontinencia urinaria en 12 mujeres $(9,5 \%)$, de las que 2 fueron intervenidas en dos ocasiones. Los tipos de cirugía antiincontinencia que se utilizaron fueron: técnica de Marshall-Marchetti-Krantz (MMK) en 7 ocasiones $(50 \%$ del total de intervenciones), técnica de Kelly $3(21,42 \%)$, técnica de Burch en 2 (14,28\%) y 1 paciente $(7,14 \%)$ presentaba antecedentes de cirugía por incontinencia que no se pudo determinar. Habían seguido tratamientos médicos para su incontinencia un total de 45 pacientes $(35,7 \%)$, de las que $40(88,89 \%)$ habían seguido tratamiento con tolterodina, 2 con cloruro de oxibutinina $(4,44 \%)$ y no pudo determinarse el tipo de fármaco en tres pacientes $(6,66 \%)$.

Un total de 54 mujeres $(42,9 \%)$ refirieron haber realizado ejercicios de Kegel como trata- 
miento de su incontinencia urinaria. Estas pacientes estuvieron realizando dichos ejercicios durante una media de 3,07 meses (DE: 4,62; IC 95\%: $1,81-4,34)$.

La media de evolución de la incontinencia urinaria fue 114,48 meses, con una mediana de 96 meses. En relación a la frecuencia miccional diurna, 76 pacientes orinaban cada 60-120 minutos $(60,3 \%), 8$ cada 30 minutos $(6,3 \%)$ y 3 cada 240 minutos $(2,4 \%)$, siendo la mínima frecuencia miccional cada 30 minutos y la máxima cada 240 . En cuanto a la frecuencia miccional nocturna, 33 pacientes se levantaban a orinar durante la noche 3 veces $(26,2 \%)$, 1 paciente lo hacía 7 veces $(0,8 \%)$ y 29 pacientes no precisaban levantarse a orinar durante la noche (23\%) (Tabla IV).

Durante la última semana, un total de 73 mujeres $(57,94 \%)$ tuvieron más de 10 episodios de escape de orina al día, mientras que únicamente 4 presentaron un solo episodio de incontinencia al día $(3,2 \%)$. En cuanto al número de compresas utilizadas diariamente, un $82,5 \%$ (104 casos) utilizaban menos de 6 compresas diarias. Urgencia miccional e incontinencia urinaria de urgencias se evidenció en 18 ocasiones respectivamente $(14,3 \%)$.
No presentaban ningún grado de cistocele 38 pacientes $(30,2 \%)$ y del resto de mujeres con cistocele, en 61 casos éste fue de grado III $(48,4 \%)$; en 25 de grado II $(19,8 \%)$ y en 2 de grado I $(1,6 \%)$. Así mismo no tenían rectocele 109 pacientes $(85,7 \%)$.

No se encontró relación entre la existencia de cistocele ni de grado de cistocele con la edad, índice de masa corporal, frecuencia miccional diurna, frecuencia miccional nocturna, número medio de escapes urinarios diarios durante la última semana ni con el número de compresas al día que utilizaban las pacientes. Tampoco se encontró asociación entre cistocele con urgencia miccional, presencia de incontinencia de urgencia, ni con antecedentes de histerectomía previa, con la edad de menarquia ni con la de menopausia.

En cuanto a la relación de la presencia de cistocele con el número de embarazos, partos y número de cesáreas, únicamente se comprobó que las mujeres con cistocele presentaron un número medio de partos vaginales superior $(3,19$ partos) a los que presentaban las mujeres sin cistocele $(2,53)$.

Sí se comprobó asociación entre la presencia de cistocele y rectocele, de forma que el 18,2\%

TABLA III. NÚMERO DE EMBARAZOS, PARTOS, CESÁREAS Y ABORTOS QUE PRESENTARON LAS PACIENTES DEL ESTUDIO.

\begin{tabular}{|l|c|c|c|c|c|}
\hline NÚMERO & $\begin{array}{c}\text { EMBARAZOS } \\
\text { № }(\%)\end{array}$ & $\begin{array}{c}\text { PARTOS } \\
\text { № }(\%)\end{array}$ & $\begin{array}{c}\text { PARTOS VAGINALES } \\
\text { № }(\%)\end{array}$ & $\begin{array}{c}\text { CESÁREAS } \\
\text { № }(\%)\end{array}$ & $\begin{array}{c}\text { ABORTOS } \\
\text { № }(\%)\end{array}$ \\
\hline 0 & $2(1,6)$ & $2(1,6)$ & $3(2,8)$ & $114(90,5)$ & $89(70,6)$ \\
\hline 1 & $5(4,0)$ & $7(5,6)$ & $10(7,9)$ & $9(7,1)$ & $24(19,0)$ \\
\hline 2 & $37(29,4)$ & $42(33,3)$ & $41(32,5)$ & $2(1,6)$ & $11(8,7)$ \\
\hline 3 & $26(20,6)$ & $33(26,2)$ & $33(26,2)$ & $1(0,8)$ & $2(1,6)$ \\
\hline 4 & $29(23,0)$ & $27(21,4)$ & $25(19,8)$ & & \\
\hline 5 & $7(5,6)$ & $7(5,6)$ & $6(4,8)$ & & \\
\hline 6 & $13(10,3)$ & $3(2,8)$ & $4(3,2)$ & & \\
\hline 7 & $3(2,8)$ & $3(2,8)$ & $2(1,6)$ & & \\
\hline 8 & $1(0,8)$ & & & & \\
\hline 9 & $3(2,8)$ & $2(1,6)$ & $2(1,6)$ & & \\
\hline
\end{tabular}


de las mujeres con algún grado de cistocele presentaban un rectocele asociado, mientras que sólo presentaron rectocele el $2,6 \%$ de las mujeres sin cistocele $(p=0,008)$.

Referían haber presentado algún episodio de infección urinaria 46 pacientes $(36,5 \%)$. La existencia de infección urinaria se asoció con la presencia de cistocele, de tal manera que presentaban antecedentes de infección urinaria un $26,3 \%$ de las mujeres sin cistocele frente a un $40,9 \%$ de las mujeres que tenían algún grado de cistocele $(p=0,05)$.

Por lo que respecta al diagnóstico urodinámico de las pacientes del estudio, en 8 casos $(6,3 \%)$ el estudio urodinámico se describió como normal, en $16(12,7 \%)$ fueron diagnosticadas de incontinencia urinaria mixta $y$, finalmente, $102(81 \%)$ fueron catalogadas como portadoras de incontinencia genuina de esfuerzo. El diagnóstico urodinámico no se asoció con la presencia de cistocele ni con la existencia de histerectomía previa.

El riesgo quirúrgico de las pacientes sometidas a cirugía de incontinencia urinaria valorado mediante la Clasificación de la Sociedad Americana de Anestesiología, fue: ASA I en 23 ocasiones (18,3\%), ASA II en 87 casos (69\%) y ASA III en $16(12,7 \%)$.
La edad media de las pacientes fue mayor en las que presentaron riesgo anestésico ASA II ० III $(58,27$ años -DE: 9,7- y 58,92 años -DE: 8,7-, respectivamente), en comparación con las que tenían riesgo ASA I (51,31 años; DE: 7,26) $(p=0,009)$.

Se comprobó una tendencia lineal significativa entre el número de problemas de salud y el riesgo anestésico ASA y con el número de fármacos utilizados $(p<0,0001)$. La media de IMC fue superior en pacientes ASA III $(p=0,021)$.

El tipo de cirugía antiicontinencia más frecuentemente realizado fue la uretropexia mediante cabestrillo transobturatriz (TOT) que se practicó en 63 casos $(50 \%)$, seguida de la uretropexia con anclaje óseo mediante "sling In Fast" en $48(38,1 \%)$ y de la técnica mediante "Tensión-free Vaginal Tape" (TVT) en $15(11,9 \%)$.

Se realizaron, además de la técnica principal de incontinencia, un total 12 tipos de cirugías asociadas a ésta, que consistieron en: 6 colporrafias posteriores y una colporrafia anterior.

Al relacionar el tipo de cirugía con las distintas variables del estudio, únicamente se encontró que presentaron enfermedades crónicas asociadas el

TABLA IV. CLIINICA MICCIONAL DE LAS PACIENTES DEL ESTUDIO.

\begin{tabular}{|c|c|c|c|c|c|c|}
\hline \multicolumn{2}{|c|}{$\begin{array}{c}\text { FRECUENCIA MICCIONAL } \\
\text { DIURNA* }^{*} \mathrm{n}^{\circ}(\%)\end{array}$} & \multicolumn{2}{|c|}{$\begin{array}{c}\text { FRECUENCIA MICCIONAL } \\
\text { NOCTURNA** }^{*}{ }^{\circ}(\%)\end{array}$} & \multicolumn{2}{|c|}{$\begin{array}{c}\text { NÚMERO DE ESCAPES } \\
\text { DIARIOS*** no (\%) }\end{array}$} & $\begin{array}{c}\text { NÚMERO DE COMPRESAS } \\
\text { DIARIAS*** no (\%) }\end{array}$ \\
\hline Cada $30^{\prime}$ & $8(6,3)$ & 0 & $29(23,0)$ & $1-2$ & $8(6,4)$ & $1 \quad 12(9,5)$ \\
\hline Cada 45' & $2(1,6)$ & 1 & $24(19,0)$ & $3-4$ & $12(9,6)$ & $2 \quad 16(12,7)$ \\
\hline Cada 60' & $30(23,8)$ & 2 & $33(26,2)$ & $5-6$ & $24(19,0)$ & $324(19,0)$ \\
\hline Cada 90' & $6(4,8)$ & 3 & $22(17,5)$ & $7-8$ & $9(7,2)$ & $420(15,0)$ \\
\hline Cada 120' & $40(31,7)$ & 4 & $8(6,3)$ & $10-12$ & $35(27,8)$ & $5 \quad 18(14,3)$ \\
\hline Cada 130' & $1(0,8)$ & 5 & $5(4,0)$ & $15-16$ & $19(15,1)$ & $6 \quad 14(11,1)$ \\
\hline Cada 150' & $15(11,9)$ & 6 & $4(3,2)$ & $20-30$ & $19(15,1)$ & $5(4,0)$ \\
\hline Cada 180' & $17(15,3)$ & 7 & $1(0,8)$ & & & $8-9 \quad 8(6,4)$ \\
\hline Cada $210^{\prime}$ & $4(3,2)$ & & & & & $107(5,6)$ \\
\hline Cada 240' & $3(2,4)$ & & & & & $2(1,6)$ \\
\hline
\end{tabular}


$81,3 \%$ de las enfermas intervenidas mediante técnica "In Fast", el $60 \%$ de las que se sometieron a un TVT y el $58,1 \%$ de las intervenidas mediante TOT $(p=0,003)$.

Presentaron complicaciones peroperatorias 6 pacientes $(4,8 \%)$. Las complicaciones peroperatorias fueron: 5 casos de apertura vesical y 1 de hemorragia vaginal con posterior formación de un hematoma pélvico.

Por lo que atañe a la existencia de complicaciones postoperatorias inmediatas, éstas se produjeron en 5 casos $(4,0 \%)$. La única complicación postoperatoria que apareció fue la existencia de retención urinaria, que obligó a portar la sonda vesical durante más de 2 días.

Finalmente, 13 pacientes $(10,3 \%)$ presentaron complicaciones tardías relacionadas con su incontinencia: 8 casos de rechazo del cabestrillo utilizado, 4 de urgencia miccional que requirió trata- miento con anticolinérgicos y 1 caso de intrusión del cabestrillo en uretra. La Tabla $V$ muestra las complicaciones encontradas según tipo de intervención y el porcentaje de éxitos.

No hubo diferencias en cuanto a la incidencia de complicaciones peroperatorias o postoperatorias en relación a la edad de las pacientes, características sociodemográficas, antecedentes de morbilidad asociada y consumo de medicación y datos de exploración física ni tipo de intervención realizada.

Sí se demostró en el análisis bivariante que la existencia de complicaciones peroperatorias se asoció con una edad de histerectomía mayor y un IMC menor. En lo referente a la existencia de complicaciones postoperatorias, la presencia de complicaciones postoperatorias inmediatas se asoció con un menor número de partos previos, una edad de menarquia mayor, un número menor de compresas/día. Finalmente, la existencia de complicaciones postope-

TABLA V. COMPLICACIONES RELACIONADAS CON LA INTERVENCIÓN Y PORCENTAJE DE ÉXITOS.

\begin{tabular}{|c|c|c|c|c|}
\hline & $\begin{array}{l}\text { COMPLICACIONES } \\
\text { PEROPERATORIAS }\end{array}$ & $\begin{array}{c}\text { COMPLICACIONES } \\
\text { POSTOPERATORIAS } \\
\text { INMEDIATAS }\end{array}$ & $\begin{array}{c}\text { COMPLICACIONES } \\
\text { POSTOPERATORIAS TARDIAS }\end{array}$ & $\begin{array}{c}\% \text { DE EXITOS } \\
\text { (PACIENTES } \\
\text { SECAS) }\end{array}$ \\
\hline $\begin{array}{l}\text { "IN } \\
\text { FAST" } \\
(n=48)\end{array}$ & $\begin{array}{c}2 \text { apertura vesical } \\
\qquad(4,16 \%) \\
\text { 1hematoma pélvico } \\
(2,08 \%)\end{array}$ & $\begin{array}{c}2 \text { retención de orina } \\
\qquad(4,16 \%)\end{array}$ & $\begin{array}{l}5 \text { rechazos de malla }(10,41 \%) \\
2 \text { urgencia miccional }(4,16 \%)\end{array}$ & $70,8 \%$ \\
\hline $\begin{array}{l}\text { "TVT" } \\
(n=15)\end{array}$ & $\begin{array}{l}1 \text { apertura vesical } \\
\qquad(6,66 \%)\end{array}$ & & 1 urgencia miccional $(6,66 \%)$ & $73,3 \%$ \\
\hline $\begin{array}{l}\text { "TOT" } \\
(\mathrm{n}=62)\end{array}$ & $\begin{array}{c}2 \text { apertura vesical } \\
\qquad(3,22 \%)\end{array}$ & $\begin{array}{l}2 \text { retención de orina } \\
\qquad(3,22 \%)\end{array}$ & $\begin{array}{c}3 \text { rechazos de malla }(4,83 \%) \\
1 \text { urgencia miccional }(1,61 \%) \\
1 \text { intrusión de malla en uretra } \\
(1,61 \%)\end{array}$ & $77 \%$ \\
\hline $\begin{array}{l}\text { "KELLY" } \\
(\mathrm{n}=1)\end{array}$ & & $\begin{array}{l}1 \text { retención de orina } \\
(100 \%)\end{array}$ & & $100 \%$ \\
\hline
\end{tabular}


ratorias tardías únicamente se asoció con un menor número de meses de evolución de la incontinencia. En la Tabla VI se resumen todos estos datos.

Tras la cirugía de la incontinencia urinaria, se comprobó que la frecuencia miccional diurna fue superior a 120 minutos en 112 pacientes $(88,9 \%)$. En cuanto a la frecuencia miccional nocturna, 110 pacientes $(87,3 \%)$ se levantaban a orinar durante la noche menos de 2 veces. Por lo que respecta al número de escapes al día durante la última semana, 104 enfermas $(82,5 \%)$ presentaban menos de 2 episodios. Finalmente, tras la cirugía, 105 pacientes $(83,3 \%)$ necesitaban como mucho usar una compresa al día durante la última semana.

Aumentaron su intervalo miccional diurno 69 pacientes $(54,8 \%)$, con un aumento medio de 81,74 minutos (IC: 95\%; 73,21-90,27) ( $p<0,0001)$. Disminuyó la frecuencia miccional nocturna en 70 mujeres $(55,6 \%)$. Entre las pacientes que disminuyeron su frecuencia miccional nocturna se comprobó una disminución media de 1,76 veces (IC: 95\%; 1,54-1,97) $(p<0,0001)$.
Disminuyó el número de episodios de escapes de orina durante la última semana tras la cirugía en 114 pacientes $(90,5 \%)$, con una disminución media de 9,65 escapes (IC: 95\%; 8,56-10,79) $(p<0,0001)$. Un total de 94 pacientes $(76,4 \%)$ estaban totalmente secas tras la cirugía. De las pacientes que quedaron totalmente secas, $88 / 94(93,6 \%)$ estabas diagnosticadas en la prueba urodinámica de I.U. genuina de esfuerzo o tenían un estudio urodinámico normal. Del total de pacientes con diagnóstico urodinámico normal o de incontinencia genuina de esfuerzo $(88 / 110)$, se encontraban totalmente secas un $80,0 \%$ frente a un $37,5 \%$ de las pacientes que tenían diagnóstico urodinámico de incontinencia urinaria mixta (6/16) ( $>>0,0001)$.

Las pacientes que mantenían algún escape tras la cirugía estaban diagnosticadas de I.U. mixta en la prueba urodinámica en el 31,3\% (10/32 casos). Se encontraban totalmente secas a los 6 meses de la intervención el 70,8\% de las mujeres intervenidas mediante técnica "in fast", el $73,3 \%$ de las sometidas a técnica TVT y el $77,0 \%$ de las intervenidas mediante procedimiento TOT.

\section{TABLA VI. VARIABLES RELACIONADAS CON LA EXISTENCIA DE COMPLICACIONES PEROPERATORIAS Y POSTOPERATORIAS INMEDIATAS Y TARDÍAS}

COMPLICACIONES PEROPERATORIAS

\begin{tabular}{|c|c|c|c|}
\hline & No complicación & Sí complicación & \multirow[b]{2}{*}{$\mathrm{p}$} \\
\hline & Media (DE) & Media (DE) & \\
\hline Edad de histerectomía & $43,37(12,05)$ & $50,0(8,48)$ & 0,043 \\
\hline Índice Masa Corporal & $28,28(4,6)$ & $25,16(4,8)$ & 0,037 \\
\hline \multicolumn{4}{|c|}{ COMPLICACIONES POSTOPERATORIAS INMEDIATAS } \\
\hline & No complicación & Sí complicación & \\
\hline & Media (DE) & Media (DE) & $\mathrm{p}$ \\
\hline Número de partos & $3,15(1,5)$ & $1,80(1,09)$ & 0,044 \\
\hline Edad de la menarquia & $12,87(1,53)$ & $14,40(1,67)$ & 0,048 \\
\hline Número de compresas/día & $4,54(2,5)$ & $2,20(1,3)$ & 0,023 \\
\hline
\end{tabular}

\section{COMPLICACIONES POSTOPERATORIAS TARDÍAS}

\begin{tabular}{c|c|c|c}
\hline & No complicación & Sí complicación & \multirow{2}{*}{$\mathrm{p}$} \\
\cline { 2 - 3 } & Media (DE) & Media (DE) & \\
\hline Meses de evolución de la incontinencia & $119,03(105,85)$ & $80,38(110,901)$ & 0,05
\end{tabular}


En cuanto al número de compresas que las pacientes necesitaban durante la última semana, 113 pacientes $(89,7 \%)$ refirieron una disminución de su número tras la cirugía. La disminución media de compresas fue de 4,09 (IC: 95\%; 3,69-4,51) (p<0,0001).

Disminuyeron la frecuencia miccional diurna tras la cirugía el $66,7 \%$ de las pacientes que habían seguido tratamiento con ejercicios de Kegel, frente al $45,8 \%$ de las pacientes que no habían seguido este tratamiento $(p=0,02)$. La media de meses bajo tratamiento con ejercicios de Kegel fue mayor en las pacientes que mejoraron la frecuencia miccional diurna $(69$ frente a 57 meses en las pacientes que no mejoraron tras la cirugía) $(p<0,025)$.

Respecto a la frecuencia miccional nocturna, la edad de las pacientes que mejoraron su frecuencia miccional nocturna tras la cirugía fue mayor $(58,85$ años) que las que no presentaron un aumento del intervalo entre micciones durante el día (54,88 años) $(p=0,02)$. Por lo que respecta al índice de masa corporal, éste fue significativamente más alto en las mujeres que aumentaron su frecuencia miccional nocturna tras la cirugía $(28,97$, frente a $27,1 \mathrm{Kg} / \mathrm{m} 2$, en las que no mejoraron) ( $p=0,038)$.

La disminución en cuanto al número de episodios de escapes al día durante la última semana tras la cirugía se asoció con:

- Un menor número de partos $(p=0,01)$.

- Menor número de partos vía vaginal $(p=0,05)$.

- Haber realizado ejercicios de Kegel antes de la cirugía ( $p=0,049)$.

- En pacientes con cistocele de mayor grado $(p<0,0001)$.

TABLA VII. VARIABLES RELACIONADAS CON LA MEJORÍA EN EL NÚMERO DE EPISODIOS DE ESCAPES AL DÍA DURANTE LA ÚLTIMA SEMANA TRAS LA CIRUGÍA.

\begin{tabular}{|ccc|}
\hline & MEJORÍA & NO MEJORÍA \\
& Media $($ DE) & Media (DE)) \\
\hline EDAD & $56,58(9,74)$ & $61,92(6,27)$ \\
\hline MESES DE EVOLUCIÓN DE LA INCONTINENCIA & $107,47(96,30)$ & $181,00(62,57)$ \\
\hline NÚMERO DE PARTOS & $2,98(1,4)$ & $4,17(1,58)$ \\
\hline PARTOS VÍA VAGINAL & $2,91(1,50)$ & $3,75(1,70)$ \\
\hline EJERCICIOS DE KEGEL & $\%$ & $\%$ \\
\hline Sí & & 3,7 \\
No & 96,3 & 13,9 \\
\hline CISTOCELE & 86,1 & 100,0 \\
Grado I & & 16,0 \\
Grado II & 0,0 & 3,3 \\
Grado III & 84,0 & 7,4 \\
\hline Sí & 96,7 & \\
\hline No & 92,6 & \\
\hline
\end{tabular}


- En pacientes sin urgencia miccional asociada $(p=0,047)$.

- Una edad menor de las pacientes $(p=0,017)$.

- Un menor tiempo de evolución de la incontinencia $(p=0,021)$.

En la Tabla VII, se exponen estos datos.

\section{DISCUSIÓN}

Los episodios de escapes urinarios disminuyeron significativamente tras la cirugía de la IUE, encontrándose totalmente secas un $76,4 \%$ de las pacientes. Este porcentaje alcanzó un $93,6 \%$ en las mujeres que tenían un estudio urodinámico normal, mientras que de las pacientes que mantenían algún tipo de escape tras la cirugía, el $31,2 \%$ estaban diagnosticadas urodinámicamente de incontinencia urinaria mixta. Dicho de otro modo, de las 16 pacientes con diagnóstico urodinámico previo de incontinencia urinaria mixta, únicamente 6 se consideraron curadas tras la cirugía $6(37,5 \%)$, lo cual obliga a realizar una evaluación prequirúrgica mucho más exhaustiva a este tipo de pacientes. En definitiva, los síntomas que mejoran en mayor medida tras la intervención son la frecuencia miccional diurna y nocturna; es decir, las pacientes orinan menos veces de día y se levantan menos veces de noche, disminuyendo el número de escapes al día y por tanto necesitando menor número de compresas.

La edad de las pacientes influyó en los resultados, de manera que a una menor edad, los escapes de orina al día tras la cirugía mejoraron en mayor medida. Cierto es que a conforme la edad de las pacientes aumenta, también lo hace el porcentaje de incontinencia de orina, la justificación es la deprivación estrogénica que ocurre con el envejecimiento, que conducen a una atrofia generalizada de todos los músculos, incluyendo la musculatura pélvica y vesicouretral (15). Así, las pacientes más jóvenes poseen un suelo pélvico en mejor disposición estructural para responder a la intervención. La mayor mejoría de los resultados en relación a un menor tiempo de evolución de la incontinencia urinaria, puede ir en consonancia a que estas pacientes son por lo general más jóvenes y en mejores condiciones anatómicas de su suelo pélvico.

En contra de lo que cabía esperar, la obesidad de nuestras pacientes no influyó a la hora de los resultados. En la literatura consultada existen distintas opiniones, unos a favor de la no diferencia de los resultados entre obesas y no obesas $(16,17)$ y otros informan que la obesidad produce el doble de fracasos quirúrgicos (18).
La disminución en cuanto al número de episodios de escapes al día durante la ultima semana tras la cirugía se asoció con un menor número de partos y sobre todo un menor número de partos vaginales. En la literatura encontramos referencias sobre un porcentaje mayor de incontinentes entre las mujeres que habían tenido partos vaginales (19-22) que entre aquellas que no habían tenido ninguno. El parto supone un trauma para el suelo pélvico produciendo una afectación de los mecanismos de soporte vesicouretrales (23). Por tanto es lógico que un mayor número de partos, sobre todo por vía vaginal, se produzca una mayor incidencia de incontinentes. Tras la cirugía se produjo una mayor mejoría en las pacientes con un menor número de partos

También se observó una disminución del número de episodios de escapes tras la cirugía en las pacientes que habían realizado previamente ejercicios de Kegel. Igualmente un fortalecimiento de la musculatura del suelo pélvico mediante estos ejercicios puede contribuir a mejorar los resultados de la cirugía de la incontinencia, puesto que el suelo pélvico está más fortalecido y en mejores condiciones de elasticidad para responder ante dicha cirugía.

Las mujeres con cistocele de mayor grado de cistocele disminuyeron en mayor medida el número de escapes de orina al día tras la cirugía. Así mismo, la presencia de cistocele en cualquier grado se asoció con la existencia de infección urinaria en un $40,9 \%$ de casos. Es sabido, y así esta descrito, que las alteraciones en el vaciado total de la vejiga, como ocurre en los cistoceles, facilitan la infección urinaria al favorecer la orina residual la multiplicación bacteriana (24). Así, al corregir el cistocele con la intervención, estamos solucionando los episodios de cistitis como consecuencia de éste y por tanto es lógico que las pacientes con dicho prolapso evolucionen hacia un menor número de escapes de orina al día tras la cirugía.

Según las distintas técnicas empleadas, los porcentajes de curación (totalmente secas) a los 6 meses de la intervención se reparten de la siguiente manera: $70,8 \%$ de las mujeres intervenidas mediante la técnica "in fast", un 73,3\% para las operadas mediante técnica TVT y un $77,0 \%$ de las sometidas a procedimiento de TOT. Como se puede apreciar los éxitos con esta última técnica son ligeramente superiores. Estos porcentajes son similares a los consultados en la literatura. Así pues con la técnica de "In Fast", la cual fue introducida por Leach en 1996 (25), los resultados son del $100 \%$ de éxito tras la cirugía en el artículo publicado por Martínez Bengoechea et al (26). Otros estudios informan de un porcentaje de curación del $91 \%$ (27). Nuestros resultados con 
esta técnica están por debajo de la literatura con un $70,8 \%$ de éxitos, si bien es cierto que existen pocos datos publicados referentes a este procedimiento.

Con respecto a la técnica de TVT que comenzó Ulmsten en 1996 (8), se han publicado múltiples estudios, los cuales reflejan variedad de resultados exitosos Así, tenemos un estudio que relata el 100\% de éxitos (28), pero la gran mayoría de trabajos consultados presentan distintos resultados que están entre el $65 \%$ y $95 \%$ de éxitos $(28-46)$. Nuestros resultados con la técnica TVT son concordantes con la literatura consultada, siendo esta técnica la que más trabajos tiene publicados, quizás porque ha sido muy llevada a cabo también por ginecólogos.

Finalmente, según la técnica de TOT introducida por Delorme a comienzos de 2001 (9), se obtienen unos resultados parecidos, con buenos resultados entre el $80 \%$ y $95 \%$ de los casos $(32,47,48)$. Nuestras pacientes operadas con esta técnica están secas en un $77 \%$, porcentaje muy similar al de la literatura. Existen pocas publicaciones de esta técnica ya que hay tener en cuenta que es una técnica novedosa, a la que conforme va pasando el tiempo se van sumando cada vez más cirujanos por ser una técnica con menos complicaciones quirúrgicas a efectos de perforación vesical.

\section{CONCLUSIÓN}

La disminución en cuanto al número de escapes de orina al día tras la cirugía con técnicas de cabestrillo transvaginal es mayor en las mujeres con antecedentes de un menor número de partos, en las que habían realizado ejercicios de Kegel, en las que presentaban un cistocele en mayor grado, cuando no existía urgencia miccional asociada, en las pacientes de menor edad y con menor tiempo de evolución de su incontinencia. Tras este tipo de cirugía de la incontinencia urinaria se produce una mejoría en la clínica miccional de las pacientes, mejorando su frecuencia miccional diurna y nocturna, disminuyendo el número de escapes y de compresas utilizadas al día.

\section{BIBLIOGRAFÍA Y LECTURAS RECOMENDADAS (*lectura de interés $y^{* *}$ lectura fundamental)}

1. BATES, P.; BRADLEY, W.E.; GLEN, E. y cols.: "The standardization of terminology of lower urinary tract function". J. Urol., 12: 551, 1979.

2. ABRAMS, P.; CARDOZO, L.; FALL, M. y cols.: "The standardisation of terminology in lower urinary tract function: report from the standardization sub-committee of the International Continence Society". Urology, 6: 37, 2003.

3. WILLIAM, R.; LENDERKING, J.F. y cols.: "A review of the quality-of-life aspects of urinary urge incontinence". Pharmaco Economics, 9: 11, 1996.

*4. NIETO BLANCO, E.; CAMACHO PÉREZ, J.; DÁVILA ÁLVAREZ, V. y cols.: "Epidemiología e impacto de la incontinencia urinaria en mujeres de 40 a 65 años de edad en un área sanitaria de Madrid". Aten. Primaria, 32: 410, 2003.

5. ABRAMS, P.; CARDOZO, L.; FALL, M. y cols.: "The standardization of terminology of lower urinary tract function: Report from the Standardization Sub-committee of the International Continence Society". Neurourol. Urodyn., 21: 167, 2002.

*6. SOLER ROSELLÓ, A.; VILA BARJA, J.; REIG RUIZ, J.: "Incontinencia urinaria femenina". Tratado de Urología, Jiménez Cruz J.F. y Rioja Sanz L.A. J.R Proas, S.A. Barcelona, 1663, 1993.

**7. McGUIRE, E.J.; LYTTON, B.: "Pubovaginal sling procedure for stress incontinence". J. Urol., 119: 82, 1978.

**8. ULMSTEN, U.; HENRIKSSON, L.; JOHNSON, P. y cols.: "An ambulatory surgical procedure under local anesthesia for treatment of female urinary incontinence". Int. Urogynecol. J. Pelvic. Floor Dysfunct., 7: 81, 1996

**9. DELORME, E.: “Transobturator urethral suspension: mini-invasive procedure in the treatment of stress urinary incontinence in women”. Prog. Urol., 11: 1306, 2001.

**10. DELORME, E.; DROUPY, S.; DE TAYRAC, R. y cols.: "Transobturator tape (uratape). A new minimally invasive method in the treatment of urinary incontinence in women”. Prog. Urol., 13: 656, 2003.

*11. CHOE, J.M.: "Suprapubic sling adjustment: minimally invasive method of curing recurrent stress incontinence after sling surgery”. J. Urol., 168: 2059, 2002.

**12. LEACH, G.E.: "Bone fixation technique for transvaginal needle suspension”. Urology, 31: 388, 1988.

*13. NATIV, O.; LEVINE, S.; MADJAR, S. y cols.: "Incisionless per vaginal bone anchor cystourethropexy for the treatment of female stress incontinence: experience with the first 50 patients". J. Urol., 158: 1742, 1997.

14. FERDINAND, F.; LITTLEJOHN, J.: "Células madre e incontinencia urinaria femenina". Biblioteca de salud de healthfinder, 2005.

**15. SALINAS CASADO, J.; VIRSEDA CHAMORRO, M.; TEBA DEL PINO, F. y cols.: "Urinay incontinence in menopause". Arch. Esp. Urol., 53: 349, 2000.

*16. LOVATSIS, D.; GUPTA, C.; DEAN, E. y cols.: "Tension-free vaginal tape procedure is an ideal treatment for obese patients". Am. J. Obstet. Gynecol., 189: 1601, 2003.

*17. RAFII, A.; DARAI, E.; HAAB, F. y cols.: "Body mass index and outcome of tension-free vaginal tape”. Eur. Urol., 43: 288, 2003.

*18. RAMÍREZ MELGAR, E.; IRIS DE LA CRUZ, S.; MARTÍNEZ GONZÁLEZ, L. y cols.: "Obesity as a risk factor in surgery for urinary incontinence". Gi- 
necol. Obstet. Mex., 65: 458, 1997.

19. PEYRAT, L.; HAILLOT, O.; BRUYERE, F. y cols.: "Prevalence and risk factors of urinary incontinence in young women". Prog. Urol., 12: 52, 2002.

20. SHAKHATREH, F.M.: "Epidemiology of urinary incontinence in Jordanian women". Saudi Med. J., 26: 830, 2005.

*21. GOLDBERG, R.P.; KWON, C.; GANDHI, S. y cols.: "Urinary incontinence after multiple gestation and delivery: impact on quality of life". Int. Urogynecol. J. Pelvic Floor Dysfunct., 16: 334, 2005.

*22. DOLAN, L.M.; WALSH, D.; HAMILTON, S. y cols.: "A study of quality of life in primigravidae with urinary incontinence". Int. Urogynecol. J. Pelvic Floor Dysfunct., 15: 160, 2004.

23. RAZ, S.; LITTLE, N.A.; JUMA, S.: "Urología femenina”. Walsh Campbell Urología, 75: 2736, 1992.

24. MURILLO MIRAT, J.; CUERVO PINNA, C.; RODRÍGUEZ RINCÓN, J.: "Infecciones urinarias no complicadas". Urología: Libro del Residente. L. Resel Estévez. Madrid, 377, 1998.

*25. LEACH, G.E.; APPELL, R.: "Percutaneous bladder neck suspension". Urol. Clin. North Am., 23: 511, 1996.

*26. MARTÍNEZ BENGOECHEA, J.J.; MILLÁN SERRANO, J.A.; MARTÍNEZ LASIERRA, M. y cols.: "Uretrocistopexia con anclajes óseos: modificación técnica". Actas Urol. Esp., 25: 20, 2001.

*27. FRANCO, N.; SHOBEIRI, S.A.; ECHOLS, K.T.: "Medium-term follow-up of transvaginal suburethral slings: variance in outcome success using two different evaluation methods". Urology, 60: 607, 2002.

*28. NAVALÓN VERDEJO, P.; ZARAGOZA FERNÁNDEZ, C.; ORDONO DOMÍNGUEZ, F. y cols.: “Tratamiento quirúrgico ambulatorio de la incontinencia urinaria de esfuerzo femenina". Arch. Esp. Urol., 58: 915, 2005.

*29. BOURRAT, M.; ARMAND, C.; SEFFERT, P. y cols.: "Complications and medium-term functional results of TVT in stress urinary incontinence". Prog. Urol., 13: 1358, 2003.

*30. QUICIOS DORADO, C.; FERNÁNDEZ FERNÁNDEZ, E.; GÓMEZ GARCÍA, I. y cols.: "Tratamiento de la incontinencia urinaria de esfuerzo con el sistma TVT: Complicaciones en nuestros primeros $100 \mathrm{ca}-$ sos". Actas Urol. Esp., 29: 750, 2005.

*31. ALLAHDIN, S.; McKINLEY, C.; MAHMOOD, T.A. y cols.: "Tension-free vaginal tape: 162 cases in a district general hospital". J. Obstet. Gynaecol., 24: 539, 2004.

*32. MELLIER, G.; BENAYED, B.; BRETONES, S. y cols.: "Suburethral tape via the obturator route: is the TOT a simplification of the TVT?". Int. Urogynecol. J. Pelvic Floor Dysfunct., 15: 227, 2004. BRIONES MARDONES, G.; JIMÉNEZ CIDRE,

*33. M.; FERNÁNDEZ FERNÁNDEZ, E. y cols.: "Tratamiento de la incontinencia urinaria de esfuerzo femenina con el sistema TVT (Tensión Free Vaginal Tape): nuestra experiencia”. Actas Urol. Esp., 25: 423, 2001.
*34. CANIS SANCHEZ, D.; BIELSA GALI, O.; CORTADELLAS ANGEL, R. y cols.: "Resultados y complicaciones de la técnica TVT en el tratamiento de la incontinencia de esfuerzo femenina". Actas Urol. Esp., 29: 287, 2005.

*35. HUALDE ALFARO, A.; JIMÉNEZ CALVO, J.; SARMIENTO GÓMEZ, C. y cols.: "Nuestra experiencia a cinco años y medio en TVT". Actas Urol. Esp., 30: 181, 2006.

36. HOLMGREN, C.; NILSSON, S.; LANNER, L. y cols.: "Long-term results with tension-free vaginal tape on mixed and stress urinary incontinence". Obstet. Gynecol., 106: 38, 2005.

*37. PARK, S.; HONG, B.; LEE, K.S. y cols.: "Risk factors of voiding dysfunction and patient satisfaction after tension-free vaginal tape procedure". J. Korean. Med. Sci., 20: 1006, 2005.

38. LIAPIS, A.; BAKAS, P.; CHRISTOPOULOS, P. y cols.: "Tension-free vaginal tape for elderly women with stress urinary incontinence". Int. J. Gynaecol. Obstet., 92: 48, 2006.

*39. YIP, S.K.; PANG, M.W.: "Tension-free vaginal tape sling procedure for the treatment of stress urinary incontinence in Hong Kong women with and without pelvic organ prolapse: 1-year outcome study". Hong Kong Med. J., 12: 15, 2006.

*40. JACOB, F.; SOYEUR, L.; ADHOUTE, F. y cols.: "Evaluation of the results of TVT in a series of 29 major sphincter incompetence". Prog. Urol., 13: 98, 2003.

*41. NILSSON, C.G.; FALCONER, C.; REZAPOUR, M.: "Seven-year follow-up of the tension-free vaginal tape procedure for treatment of urinary incontinence”. Obstet. Gynecol., 104: 1259, 2004.

42. RICHTER, H.E.; NORMAN, A.M.; BURGIO, K.L. y cols.: "Tension-free vaginal tape: a prospective subjective and objective outcome analysis". Int. Urogynecol. J. Pelvic Floor Dysfunct., 16: 109, 2005.

*43. MESCHIA, M.; PIFAROTTI, P.; BUONAGUIDI, A. y cols.: "Tension-free vaginal tape (TVT) for treatment of stress urinary incontinence in women with low-pressure urethra". Eur. J. Obstet. Gynecol. Reprod. Biol., 122: 118, 2005.

*44. HERMIEU, J.F.: "Bandelettes sous-urétrales dans l'incontinence urinaire d'effort de la femme: revue des différents procédés". Ann. Urol., 39: 124, 2005.

*45. MORÁN, P.A.; WARD, K.L.; JOHNSON, D. y cols.: "TVT para la incontinencia de esfuerzo genuina primaria: un estudio de seguimiento en dos centros". BJU, 86: 39, 2000.

46. SANZ PÉREZ, G.; RODRÍGUEZ-RUBIO CORTADILLAS, F.I. y cols.: "TVT para la incontinencia urinaria femenina: estudio multicéntrico español”. Actas Urol. Esp., 29: 632, 2005.

**47. ROUMEGUERE, T.; QUACKELS, T.; BOLLENS. R. y cols.: "Trans-obturator vaginal tape (TOT) for female stress incontinence: one year follow-up in 120 patients". Eur. Urol., 48: 805, 2005.

**48. CINDOLO, L.; SALZANO, L.; ROTA, G. y cols.: "Tension-free transobturator approach for female stress urinary incontinence”. Minerva Urol. Nefrol., 56: 89, 2004. 